\title{
Consumption of preserved egg, a high-lead-containing food, is strongly associated with depressive symptoms in Chinese adults
}

\author{
Bin $\mathrm{Yu}^{1,2}$, Junwei Shao ${ }^{1}$, Fei Yu ${ }^{1}$, Qing Zhang ${ }^{3}$, $\mathrm{Li} \mathrm{Liu}^{3}$, Ge Meng ${ }^{1}$, Hongmei Wu ${ }^{1}$, Yang Xia ${ }^{1}$, Xue Bao ${ }^{1}$, \\ Yeqing $\mathrm{Gu}^{1}$, Hongbin $\mathrm{Shi}^{3}$, Shaomei Sun ${ }^{3}$, Xing Wang ${ }^{3}$, Ming Zhou ${ }^{3}$, Qiyu Jia ${ }^{3}$, Huijun Liu $^{2}$, \\ Yuntang $\mathrm{Wu}^{1 *}$ and Kaijun $\mathrm{Niu}^{1,3 *}$ \\ ${ }^{1}$ Nutritional Epidemiology Institute and School of Public Health, Tianjin Medical University, Tianjin, 300070 People's \\ Republic of China \\ ${ }^{2}$ Institute of Psychology, Tianjin Medical University, Tianjin, 300070 People's Republic of China \\ ${ }^{3}$ Health Management Centre, Tianjin Medical University General Hospital, Tianjin, 300052 People's Republic of China
}

(Submitted 14 July 2017 - Final revision received 6 September 2017 - Accepted 8 September 2017)

\begin{abstract}
Previous studies have demonstrated adverse mental health effects of $\mathrm{Pb}$ exposure. The purpose of this study is to investigate the relationship between consumption of preserved egg (PE), a high-Pb-containing food and depressive symptoms among adults in China. A sample of 25213 adults (mean age 41.4 (sD 11.8) years; males, 53.9\%) in Tianjin, China, was studied in a cross-sectional analysis. Dietary intake including PE was assessed using a valid self-administered FFQ. Depressive symptoms were assessed using the Self-Rating Depression Scale (SDS). The association was estimated by OR using logistic regression models adjusted for multiple confounders. The prevalence of elevated depressive symptoms was $6 \cdot 6 \%$ (SDS $\geq 50$ ). Compared with the least frequent PE consumption (<once/week), multivariable adjusted OR for elevated depressive symptoms were 1.52 (95\% CI 1.28, 1.81), $2 \cdot 24$ (95\% CI 1.76, 2.81) and 3.31 (95\% CI 2.52, 4.30) for consumption of once, 2-3 times and $\geq 4$ times/week, respectively $\left(P_{\text {for trend }}<0 \cdot 0001\right.$ ), indicating a clear dose-response relationship. The results suggested that higher consumption of PE was strongly associated with depressive symptoms among adults in China. These findings underscore the need to consider dietary $\mathrm{Pb}$ exposure as a risk factor for psychological distress.
\end{abstract}

\section{Key words: Preserved eggs: Lead: Depressive symptoms: Cross-sectional studies}

$\mathrm{Pb}$ is a well-known neurotoxicant that is pervasive in the environment, found in air, soil and water. Developed countries have tried and succeeded in reducing the level of exposure to $\mathrm{Pb}$ since $1980^{(1)}$. The Chinese government began to ban the use of leaded gasoline nationally since 1 July 2000, which also decreased the $\mathrm{Pb}$ levels in air and soil. However, several other sources of $\mathrm{Pb}$ exposure remain for Chinese people, one of which is dietary.

Preserved egg (PE), also known as Pidan (Chinese: 皮蛋; pinyin: pídàn), is a Chinese preserved food product and delicacy made by preserving duck or chicken eggs in a mixture of clay, ash, salt, quicklime and rice hulls for several weeks to several months. The nutritional value of PE is slightly decreased compared with that of fresh egg, but PE has an extremely long shelf life and a pleasant, fragrant taste that is preferred by most people in Southeast Asian countries ${ }^{(2)}$. In particular, PE usually contains quite a high amount of $\mathrm{Pb}$ because of the use of litharge powder $(\mathrm{PbO})$ in pickle processing. $\mathrm{PbO}$ can penetrate through the eggshell and increase the $\mathrm{Pb}$ level in eggs.
According to an investigation through the national food contamination monitoring system, the average $\mathrm{Pb}$ content of $\mathrm{PE}$ consumed in China was $1.782 \mathrm{mg} / \mathrm{kg}$, with the maximum of $334.0 \mathrm{mg} / \mathrm{kg}$, which is much higher than any other commonly consumed food $^{(3)}$. Recently, another study showed that among all Chinese food groups the highest $\mathrm{Pb}$ exposure was from 'Eggs and their products' (42.4-51.6\% of the total exposure), with PE as the main contributor ${ }^{(4)}$.

Various adverse effects of $\mathrm{Pb}$ exposure on human health have been established. Disorders of the gastrointestinal, renal, hepatic, haematologic and immunologic systems, as well as cardiovascular systems, have been reported ${ }^{(5,6)}$. Neurotoxicity is a well-known clinical feature of $\mathrm{Pb}$ exposure. Consistent neuropsychological research has revealed that $\mathrm{Pb}$ exposure can result in declines in intelligence, memory, comprehension and reading, visuospatial skills and executive functions ${ }^{(7)}$. Adverse psychological symptoms also emerged as a result of $\mathrm{Pb}$ exposure. Studies on the mental health effects of $\mathrm{Pb}$ exposure were mainly in the context of occupational exposures, with levels of

Abbreviations: PA, physical activity; PE, preserved egg; SDS, Self-Rating Depression Scale.

* Corresponding authors: K. Niu, email nkj0809@gmail.com; Y. Wu, email niukaijun@tmu.edu.cn 
exposure much higher than that experienced by the general population. Pb-exposed workers in foundries, battery plants and $\mathrm{Pb}$ smelters were reported to experience mood disorders such as anxiety, hostility and depressive states ${ }^{(8,9)}$. Only one study has examined the relationship between environmental $\mathrm{Pb}$ exposure and depression in general populations, which did not demonstrate a consistent association within the investigated blood $\mathrm{Pb}$ levels ${ }^{(10)}$.

For the population at large, which is not occupationally exposed, food and water are the most important sources of exposure to $\mathrm{Pb}^{(11)}$. The health impact of long-term $\mathrm{Pb}$ exposure through dietary intake, which may cause adverse health effects even at relatively low levels, has attracted researchers' attention in recent years ${ }^{(12,13)}$. However, few studies have considered the possible link between dietary $\mathrm{Pb}$ exposures and mental health outcomes in the general population. Given that dietary $\mathrm{Pb}$ exposure is relatively modifiable, this type of potential risk factor for mental health outcomes should be of interest. As a single food with a high content of $\mathrm{Pb}, \mathrm{PE}$ is quite a preferred candidate for investigating the relationship between dietary $\mathrm{Pb}$ exposure and mental health. Therefore, we turned to the comprehensive physical examination data collected in Tianjin to explore the association between consumption of $\mathrm{PE}$ and depressive symptoms in a large population of Chinese adults.

\section{Methods}

\section{Study participants}

This analysis used data from the Tianjin Chronic Low-grade Systemic Inflammation and Health Cohort, which is a large prospective cohort study of inhabitants living in Tianjin, China. The cohort was based on annual health examinations conducted in Tianjin Medical University General Hospital Health Management Center and focused on the relationship between chronic low-grade systemic inflammation and the health status. Participants who had received physical examinations and completed a detailed lifestyle questionnaire were recruited (92.3\% response rate). Recruitment took place from May 2013 to December 2016. Written informed consent was obtained for every participant, and the Institutional Review Board of Tianjin Medical University approved study protocols.

During the study period, a total of 27946 participants aged 20 years and older were sampled. Participants who did not complete data collection on FFQ or depression scale ( $n$ 974), BMI and physical activity (PA) ( $n$ 139), or those with a history of CVD ( $n$ 1343) or cancer ( $n$ 277), were excluded. Thus, a final sample of 25213 participants (mean age 41.4 (SD 11.8) years; males, $53.9 \%$ ) was used in current analyses.

\section{Measures}

Assessment of dietary intake. Dietary intake during the previous month was obtained through a validated FFQ that included 100 food items with specified serving sizes. The FFQ included seven frequency categories ranging from 'never' to ' 2 or more times/d' for foods (including PE and egg) and eight frequency categories ranging from 'never' to ' 4 or more times/d' for beverages. By combining the information obtained from the food frequency response with the Chinese food composition tables $^{(14)}$, we were able to compute the mean total energy intake. The FFQ have been evaluated with regard to reproducibility and validity ${ }^{(15)}$

Assessment of depressive symptoms. Depressive symptoms were assessed by the Chinese version of Self-Rating Depression Scale (SDS), which has been proven to be a valid measure for the Chinese population ${ }^{(16)}$. There are twenty items rated on a four-point scale. Summary scores range from 20 to 80, with higher scores indicating the elevated depressive symptoms. In order to increase sensitivity, two cutoff points ( 45 and 50) were used to define depressive symptoms in the present study ${ }^{(17,18)}$. Scores higher than these cutoff points are considered to reflect moderately or severely depressive symptoms. Continuous scores of SDS were also used in the sensitivity analyses.

Assessment of other variables. Participants completed standardised physical examinations at the Health Management Centers, which included measurements of height, weight, waist circumference, blood pressure, fasting blood sugar and plasma lipids ${ }^{(15,19)}$. BMI was calculated as weight (kg) divided by height (m) squared. The metabolic syndrome (MetS) was defined according to the criteria of the American Heart Association Scientific Statement ${ }^{(20)}$.

Socio-demographic variables including sex, age, education, occupation, household income and social connections (including marital status, cohabitants and amount of social contact) were also assessed. Smoking and drinking status was obtained from the questionnaire. PA in the most recent week was assessed using the short version of the International Physical Activity Questionnaire ${ }^{(21)}$. For evaluation of total PA, separate metabolic equivalent (MET) hours per week were calculated for walking, moderate and vigorous activities (MET coefficient for each category was $3.3,4.0$ and 8.0 , respectively) according to the following formula: MET coefficient of activity $\times$ duration $(h) \times$ frequency $(\mathrm{d})^{(21)}$. Total PA level was calculated by summing scores for different activities.

\section{Statistical analysis}

Descriptive data are presented as the means and 95\% CI or percentages. Four categories of PE consumption were used to classify the participants: <once/week, once/week, 2-3 times/ week and $\geq 4$ times/week. Differences in covariates between the PE consumption categories were examined by ANOVA for continuous variables or by logistic regression analysis for categorical variables. Depressive symptoms were analysed as binary variables using the lower $(\geq 45)$ and higher $(\geq 50)$ cutoff points. Logistic regression models were fitted to assess the associations between PE consumption categories and depressive symptoms, using the lowest category of PE consumption (<once/week) as the reference group. For all analyses, three different models were fitted: model 1 , a crude univariate model; model 2, adjusted for age, sex and BMI; and model 3, adjusted for smoking and drinking, PA, education, employment, household income, cohabitants, social contact, marital, total 
energy intake, the MetS and egg consumption. The final multivariate logistic analysis was performed with the forced entry of all factors considered to be potential covariates. The interactions between PE consumption and sex for depressive symptoms were tested through the addition of the cross-product term to the final regression model. All $P$ values for linear trends were calculated by using the categories of PE consumption. Significance level was set at $0 \cdot 05$. All the statistical analyses were performed by using SAS (version 9.1; SAS Institute).

\section{Results}

A total number of 25213 participants were included in the final analysis, with the average BMI being $24.5(\mathrm{SD} 3 \cdot 8) \mathrm{kg} / \mathrm{m}^{2}$. Of all the participants, the mean SDS score was $36 \cdot 8$, with a median of $36 \cdot 0 ; 17.0 \%$ were classified as having elevated depressive symptoms when using 45 as a cutoff point, and $6.6 \%$ with the higher cutoff point of 50 . Participant characteristics according to categories of PE consumed are presented in Table 1. Most of the participants (88.4\%) in this cohort reported consuming PE less than once/week, whereas only $2 \cdot 4 \%$ consumed PE more than 4 times/week. Compared with those with least frequent consumption of PE, the higher PE consumers were more likely to be males and younger; were less likely to be married and more likely to be living alone; and had lower household income, a lower PA level, higher BMI and higher prevalence of the MetS. PE consumption was positively associated with smoking and alcohol use, and negatively associated with egg consumption. Mean total energy intake was significantly higher across the PE consumption quartiles.

The crude and adjusted association between categories of PE consumption and depressive symptoms are shown in Table 2. In all three models, the ORs of the depressive symptoms remarkably increased across PE intake levels. Taking the cutoff point of 50 for example, age-, sex- and BMI-adjusted OR for depressive symptoms across categories of PE consumption were $1.00,1.65$ (95\% CI 1.39, 1.95), 2.51 (95\% CI 1.99, 3.13) and $3.76(95 \%$ CI $2.89,4.82)$, respectively ( $\left.P_{\text {for trend }}<0.0001\right)$, indicating a clear dose-response relationship. These results were almost unchanged when adjusted for multiple confounding factors. In the final multivariate logistic models, the adjusted OR for depressive symptoms across categories of PE consumption were 1.00, 1.52 (95\% CI 1.28, 1.81), $2 \cdot 24$ (95\% CI $1 \cdot 76,2 \cdot 81)$ and $3 \cdot 31(95 \%$ CI $2 \cdot 52,4 \cdot 30)\left(P_{\text {for trend }}<0 \cdot 0001\right)$. The prevalence of depressive symptoms was more than threefold for participants who consumed $\mathrm{PE} \geq 4$ times/week compared with those who consumed <once/week. Similar relations were observed when we used SDS $\geq 45$ as a definition of elevated depressive symptoms. We also tested whether similar results would be observed if depressive symptoms were treated as continuous variables. Mean SDS scores for four PE consumption categories were adjusted for all the covariates by using ANCOVA. Adjusted mean SDS scores across categories of PE consumption were $36 \cdot 2$ (95\% CI 34.3, 38.1), 37.3 (95\% CI 35.4, $39 \cdot 3), 38 \cdot 5$ (95\% CI 36.5, 40.7) and 39.6 (95\% CI 37.4, 41.8), respectively. The mean SDS scores significantly increased with increasing PE consumption $(P<0 \cdot 0001)$, which are comparable to the categorical analyses.
Additional sensitivity analyses were carried out to exclude the possible bias of the association. No significant interaction between PE consumption and sex was found (SDS $\geq 50$, $P_{\text {for interaction }}=0 \cdot 81$ ). As depressive status is also related to unhealthy eating habits and appetite ${ }^{(22,23)}$, a sensitivity analysis was added by excluding those who had very low (lower $2.5 \%$ ) or high (upper $2.5 \%$ ) energy intake. After this exclusion, the fully adjusted OR for depressive symptoms (SDS $\geq 50$ ) across categories of PE consumption were 1.00, 1.48 (95\% CI 1.23, 1.78 ), 2.21 (95\% CI $1.72,2.81$ ) and 3.08 (95\% CI $2.22,4.19$ ) $\left(P_{\text {for trend }}<0 \cdot 0001\right.$ ), which demonstrated a similar pattern of results.

\section{Discussion}

In this large-scale study, we have found that increasing consumption frequency of $\mathrm{PE}$ was strongly associated with a higher risk of depressive symptoms in adults in China. Compared with participants who consumed PE less than once/week, those who consumed more than 4 times/week had a more than 3 -foldhigher risk of increasing depressive symptoms ( $S D S \geq 50$ ). The associations were consistent after the adjustment for multiple confounding factors. The trend analyses support the presence of a significant increase in risk across the levels of PE consumption frequency.

To the best of our knowledge, this is the first large population study of the association between consumption of a high-Pbcontaining food and depressive symptoms. As PE is quite similar to the nutrient composition of egg $^{(2)}$, we have adjusted the egg consumption in our regression model. The results did not change after this adjustment, which makes us more confident to infer that it should be the high $\mathrm{Pb}$ contents in $\mathrm{PE}$ that contribute to the adverse mental health effect.

$\mathrm{Pb}$ can enter the body mainly through the respiratory and gastrointestinal tracts. For adults with occupational exposure, the most significant route for absorption is through the respiratory tract ${ }^{(24)}$. The association between occupational $\mathrm{Pb}$ exposure and depression, anxiety and other psychiatric illness has already been well documented ${ }^{(8,25,26)}$. The link between increased blood and bone $\mathrm{Pb}$ levels and psychological symptoms has also been investigated by several studies in the general population. A study of 526 older adults in the Normative Aging Study found that anxiety, depression and phobia were positively correlated with bone $\mathrm{Pb}$ level ${ }^{(27)}$. Another cross-sectional epidemiologic study found that higher blood $\mathrm{Pb}$ levels were associated with increased odds of major depression and panic disorders in US young adults ${ }^{(28)}$. Recently, a longitudinal study in South Korea with a large sample size found an association between mental disorders and an increased blood $\mathrm{Pb}$ level ${ }^{(29)}$. Our study added the evidence that dietary $\mathrm{Pb}$ exposure may also increase the risk of mental health problems. This is important as the absorption rates of $\mathrm{Pb}$ are varied for different ways of exposure. According to the previous study, the percentage of inhaled $\mathrm{Pb}$ reaching the bloodstream is estimated to be $30-40 \%$, whereas rates of absorption through the gastrointestinal tract for adults are $10-15 \%$ on average ${ }^{(30)}$.

We believe the findings to be biologically plausible, as growing literature elucidates the mechanisms of how exposure 
Table 1. Participant characteristics by frequency of preserved egg consumption

(Least square geometric means and $95 \%$ confidence intervals)

\begin{tabular}{|c|c|c|c|c|c|c|c|c|c|}
\hline & \multicolumn{8}{|c|}{ Frequency of preserved egg consumption } & \multirow[b]{3}{*}{$P_{\text {for trend }}{ }^{*}$} \\
\hline & \multicolumn{2}{|c|}{ <Once/week (n 22 285) } & \multicolumn{2}{|c|}{ Once/week ( $n$ 1821) } & \multicolumn{2}{|c|}{ 2-3 times/week ( $n$ 703) } & \multicolumn{2}{|c|}{$\geq 4$ times/week $(n 404)$} & \\
\hline & Mean & $95 \% \mathrm{Cl}$ & Mean & $95 \% \mathrm{Cl}$ & Mean & $95 \% \mathrm{Cl}$ & Mean & $95 \% \mathrm{Cl}$ & \\
\hline Age (years) & $40 \cdot 06$ & $39 \cdot 91,40 \cdot 2$ & 37.78 & $37.31,38 \cdot 27$ & 37.64 & $36.88,38.42$ & $39 \cdot 1$ & $38.06,40 \cdot 17$ & $<0.0001$ \\
\hline Sex (males, \%) & & $52 \cdot 61$ & & $64 \cdot 14$ & & 66.57 & & 57.18 & $<0.0001$ \\
\hline BMI $\left(\mathrm{kg} / \mathrm{m}^{2}\right)$ & $24 \cdot 19$ & $24 \cdot 14,24 \cdot 24$ & 24.69 & $24.52,24.86$ & 24.75 & $24.48,25.03$ & $24 \cdot 36$ & $24,24.73$ & $<0.0001$ \\
\hline Metabolic syndrome (yes, \%) & & 24.98 & & 27.72 & & 28.02 & & $25 \cdot 36$ & 0.03 \\
\hline Physical activity (MET-h/week) & $10 \cdot 3$ & $2 \cdot 32,10 \cdot 13$ & 9.63 & 2.2, 9.07 & 9.69 & $2.17,8.79$ & 8.42 & $2,7.4$ & $<0.01$ \\
\hline Total energy intake $(\mathrm{kJ} / \mathrm{d})$ & $8282 \cdot 14$ & $8251.81,8312.56$ & $8955 \cdot 18$ & $8841 \cdot 08,9070 \cdot 78$ & $9460 \cdot 40$ & $9267.14,9657.68$ & $10776 \cdot 27$ & $10486 \cdot 86,11073 \cdot 71$ & $<0.0001$ \\
\hline Total energy intake (kcal/d) & 1979.48 & $1972 \cdot 23,1986 \cdot 75$ & $2140 \cdot 34$ & $2113.07,2167.97$ & 2261.09 & $2214.9,2308 \cdot 24$ & 2575.59 & $2506 \cdot 42,2646 \cdot 68$ & $<0.0001$ \\
\hline SDS score & 35.77 & $35.67,35.87$ & 37.09 & $36 \cdot 73,37.45$ & 38.36 & $37.76,38.96$ & 39.41 & $38.61,40 \cdot 23$ & $<0.0001$ \\
\hline \multicolumn{10}{|l|}{ Smoking status (\%) } \\
\hline Smoker & & $19 \cdot 9$ & & $29 \cdot 6$ & & 29.9 & & $23 \cdot 1$ & $<0.0001$ \\
\hline Ex-smoker & & $5 \cdot 7$ & & $4 \cdot 3$ & & $5 \cdot 7$ & & 4.7 & 0.19 \\
\hline Non-smoker & & 74.4 & & $66 \cdot 1$ & & 64.4 & & $72 \cdot 3$ & $<0.0001$ \\
\hline \multicolumn{10}{|l|}{ Drinking status (\%) } \\
\hline Every day & & 4.8 & & 6.7 & & 7.8 & & 6.8 & $<0.0001$ \\
\hline Sometimes & & $56 \cdot 8$ & & 62.5 & & $60 \cdot 1$ & & $55 \cdot 8$ & $<0.01$ \\
\hline Ex-drinker & & 9.7 & & $8 \cdot 1$ & & 8.5 & & $7 \cdot 0$ & $<0.01$ \\
\hline Non-drinker & & $28 \cdot 7$ & & 22.7 & & 23.7 & & 30.5 & $<0.001$ \\
\hline Marital status (married, \%) & & $86 \cdot 7$ & & 83.5 & & 83.5 & & 83.9 & $<0.0001$ \\
\hline Living alone (yes, \%) & & 8.4 & & $9 \cdot 0$ & & $12 \cdot 6$ & & $10 \cdot 0$ & $<0.01$ \\
\hline Education ( $\geq$ college graduate, $\%$ ) & & $67 \cdot 1$ & & $69 \cdot 0$ & & 68.5 & & $66 \cdot 4$ & 0.33 \\
\hline \multicolumn{10}{|l|}{ Working status (\%) } \\
\hline Managers & & 43.5 & & 44.7 & & 40.7 & & $42 \cdot 8$ & 0.55 \\
\hline Professionals & & $16 \cdot 7$ & & $17 \cdot 6$ & & 19.5 & & $18 \cdot 4$ & 0.04 \\
\hline Others & & 39.8 & & $37 \cdot 6$ & & 39.8 & & 38.9 & 0.34 \\
\hline Household income ( $\geq 10000$ Yuan, \%) & & 36.5 & & $33 \cdot 3$ & & $32 \cdot 3$ & & $27 \cdot 3$ & $<0.001$ \\
\hline Visiting friend (yes, \%) & & 59.9 & & $57 \cdot 1$ & & 62.0 & & 61.5 & 0.93 \\
\hline \multicolumn{10}{|l|}{ Egg consumption (\%) } \\
\hline$<$ Once/week & & 4.3 & & 3.4 & & 8.6 & & $12 \cdot 7$ & $<0.0001$ \\
\hline Once/ week & & 3.7 & & 11.0 & & 11.3 & & 9.5 & $<0.0001$ \\
\hline 2-3 times/week & & $23 \cdot 0$ & & $22 \cdot 7$ & & 34.8 & & 13.7 & 0.25 \\
\hline$\geq 4$ times/week & & $69 \cdot 1$ & & $62 \cdot 9$ & & $45 \cdot 4$ & & $64 \cdot 2$ & $<0.0001$ \\
\hline
\end{tabular}

MET, metabolic equivalents; SDS, Self-Rating Depression Scale.

* ANOVA or logistic regression analysis. 
Table 2. Adjusted associations between frequency of preserved egg consumption and depressive symptoms (Adjusted odds ratios and $95 \%$ confidence intervals)

\begin{tabular}{|c|c|c|c|c|c|c|c|c|}
\hline & \multicolumn{7}{|c|}{ Frequency of preserved egg consumption } & \multirow[b]{3}{*}{$P_{\text {for trend }}{ }^{*}$} \\
\hline & \multirow{2}{*}{$\frac{<\text { Once/week }(n \text { 22 285) }}{\text { OR }}$} & \multicolumn{2}{|c|}{ Once/week ( $n$ 1821) } & \multicolumn{2}{|c|}{ 2-3 times/week ( $n$ 703) } & \multicolumn{2}{|c|}{$\geq 4$ times/week $(n 404)$} & \\
\hline & & OR & $95 \% \mathrm{Cl}$ & OR & $95 \% \mathrm{Cl}$ & OR & $95 \% \mathrm{Cl}$ & \\
\hline No. of depressive symptoms (SDS $\geq 45$ ) & 3543 & & 399 & & 202 & & 151 & \\
\hline Crude & Ref. & 1.48 & $1.32,1.67$ & $2 \cdot 13$ & $1 \cdot 8,2 \cdot 52$ & $3 \cdot 16$ & $2 \cdot 57,3.87$ & $<0.0001$ \\
\hline Adjusted for age, sex and BMI & Ref. & 1.53 & $1.36,1 \cdot 72$ & $2 \cdot 21$ & $1 \cdot 86,2 \cdot 6$ & $3 \cdot 20$ & $2.60,3.92$ & $<0.0001$ \\
\hline Multiple-adjusted model $†$ & Ref. & 1.43 & $1.27,1.61$ & $2 \cdot 03$ & $1.7,2.41$ & 2.94 & $2.37,3.63$ & $<0.0001$ \\
\hline No. of depressive symptoms (SDS $\geq 50$ ) & 1329 & & 168 & & 94 & & 77 & \\
\hline Crude & Ref. & 1.60 & $1.35,1.89$ & 2.43 & $1.93,3.03$ & 3.71 & $2 \cdot 86,4.76$ & $<0.0001$ \\
\hline Adjusted for age, sex and BMI & Ref. & 1.65 & $1.39,1.95$ & 2.51 & $1.99,3 \cdot 13$ & 3.76 & $2 \cdot 89,4.82$ & $<0.0001$ \\
\hline Multiple-adjusted model $\dagger$ & Ref. & 1.52 & $1.28,1.81$ & $2 \cdot 24$ & $1 \cdot 76,2 \cdot 81$ & 3.31 & $2 \cdot 52,4.30$ & $<0.0001$ \\
\hline
\end{tabular}

SDS, Self-Rating Depression Scale; Ref., referent values.

* Obtained by using multiple logistic regression analysis.

† Adjusted for age, sex, BMI, smoking, drinking, physical activity, marital status, total energy intake, household incomes, employment, education, visiting friends, living alone, metabolic syndrome and egg consumption.

to $\mathrm{Pb}$ may lead to psychological disorders including depression $^{(31)}$. For example, $\mathrm{Pb}$ exposure has been found to alter the normal physiology of dopamine and serotonin metabolism in the brain that are frequent targets for medications to treat depression ${ }^{(32,33)}$. Animal studies have also demonstrated that $\mathrm{Pb}$ exposure can disrupt the hypothalamic-pituitary-adrenal axis, leading to altered glucocorticoid and catecholaminergic signalling ${ }^{(34,35)}$, with mood disorder as a proposed consequence ${ }^{(34)}$. Moreover, $\mathrm{Pb}$ also has well-known adverse effects on the cardiovascular system ${ }^{(36,37)}$, and many cardiovascular risk factors have been found to predict depression.

An alternative explanation of the findings is that the relatively small subset of participants who had a relatively high frequency of PE consumption differed from the remainders in ways that might increase the prevalence of depressive symptoms. From the descriptive statistics of the cohort (Table 1), it appears that high PE consumption was associated with many unhealthier characteristics such as higher BMI, lower PA level and higher frequency of smoking and drinking. We addressed this issue by running models adjusting for potential depression risk factors associated with PE consumption (BMI, PA, education level, alcohol consumption, smoking, etc.). The fact that the association between PE consumption and depressive symptoms was not attenuated after these adjustments suggests that effects are unlikely to be attributed to these confounding factors. Moreover, the associations between PE consumption and two different cutoff points for depressive symptoms were tested and similar relationships were consistently observed.

The strength of our study includes the large sample size and the adjustment for a number of potential confounders. One limitation is that the depressive symptoms were assessed with a self-reported questionnaire rather than diagnostic psychiatric interviews. Total scores on the SDS do not correspond with a clinical diagnosis of depression but rather indicate the level of depressive symptoms that may be of clinical relevance. Therefore, a larger population study that uses a standardised comprehensive structured diagnostic interview should be undertaken to confirm the associations with depression. Another limitation is that this is an observational and correlational study, and therefore does not establish a causal relation between PE consumption and outcomes. Although we have adjusted statistically for many potential confounding factors, we may not have fully captured relevant aspects of those factors. Future laboratory studies are needed to confirm the mechanisms involved in the association between dietary $\mathrm{Pb}$ exposure and depression.

In summary, the present study provides support for a strong association between the consumption of $\mathrm{PE}$, a high-Pbcontaining food and the risk of depressive symptoms in Chinese adults. These findings underscore the need to consider dietary $\mathrm{Pb}$ exposure as a risk factor for psychological distress.

\section{Acknowledgements}

The authors gratefully acknowledge all the people who have participated in the study.

This study was supported by grants from the National Natural Science Foundation of China (nos 81673166, 81372118, 81372467 and 81302422), the key technologies R\&D program of Tianjin (Key Project: nos 11ZCGYSY05700, 12ZCZDSY20400, 13ZCZDSY20200 and 15YFYZSY00020), the National Science and Technology Support Program (no. 2012BAI02B02), 2012 and 2016 Chinese Nutrition Society Nutrition Research Foundation-DSM Research Fund (nos 2014-071, 2016-046 and 2016-023), the Technologies development programme of Beichen District of Tianjin (nos bcws2013-21, bcws2014-05 and 2015-SHGY-02), the technologies project of Tianjin Binhai New Area (nos 2013-02-04 and 2013-02-06), the Science Foundation of Tianjin Medical University (nos 2010KY28 and 2013KYQ24), the Key Laboratory of Public Health Safety (Fudan University), Ministry of Education (no. GW2014-5), the National Training Programs of Innovation and Entrepreneurship for Undergraduates (no. 201510062013) and the National Social Science Foundation (no. 15BSH065), China, Scholarship from China Scholarship Council.

B. Y., Y. W. and K. N. contributed to the conception and design and drafting or revising the article; B. Y., F. Y. and K. N. contributed to the analysis and interpretation of the data; B. Y., J. S., F. Y., G. M., H. W., Y. X., X. B., Y. G., H. S., Y. W. and K. N. contributed to the assembly of the data; B. Y., J. S., F. Y., Q. Z., 
L. L., G. M., H. W., Y. X., X. B., Y. G., H. S., S. S., X. W., M. Z., Q. J., H. L., Y. W. and K. N. contributed to data collection; B. Y. and $\mathrm{K}$. N. contributed to the approval of the final version of the manuscript.

None of the authors has any conflicts of interest to declare.

\section{References}

1. Muntner P, Menke A, DeSalvo KB, et al. (2005) Continued decline in blood lead levels among adults in the United States: the National Health and Nutrition Examination Surveys. Arch Intern Med 165, 2155-2161.

2. Wang J \& Fung DY (1996) Alkaline-fermented foods: a review with emphasis on pidan fermentation. Crit Rev Microbiol 22, 101-138.

3. Jiang DG, Wang ZT, Yang DJ, et al. (2008) [National continuous monitoring on the content of lead in preserved eggs from 2000 to 2006]. Zhonghua Yu Fang Yi Xue Za Zhi (Chi J Prev Med) 42, 304-306.

4. Pan L, Wang Z, Peng Z, et al. (2016) Dietary exposure to lead of adults in Shenzhen city, China. Food Addit Contam Part A Chem Anal Control Expo Risk Assess 33, 1200-1206.

5. Patrick L (2006) Lead toxicity, a review of the literature. Part 1: exposure, evaluation, and treatment. Altern Med Rev 11, 2-22.

6. Flora G, Gupta D \& Tiwari A (2012) Toxicity of lead: a review with recent updates. Interdiscip Toxicol 5, 47-58.

7. Mason LH, Harp JP \& Han DY (2014) Pb neurotoxicity: neuropsychological effects of lead toxicity. BioMed Res Int 2014, 840547 .

8. Schwartz BS, Lee BK, Bandeen-Roche K, et al. (2005) Occupational lead exposure and longitudinal decline in neurobehavioral test scores. Epidemiology 16, 106-113.

9. Maizlish NA, Parra G \& Feo O (1995) Neurobehavioural evaluation of Venezuelan workers exposed to inorganic lead. Occup Environ Med 52, 408-414.

10. Golub NI, Winters PC \& van Wijngaarden E (2010) A population-based study of blood lead levels in relation to depression in the United States. Int Arch Occup Environ Health 83, 771-777.

11. World Health Organization (1995) Environmental Health Criteria 165. International Programme on Chemical Safety. Geneva: WHO.

12. Sun JF, Wang CN, Wu YN, et al. (2011) Long-term dietary exposure to lead of the population of Jiangsu Province, China. Food Addit Contam Part A Chem Anal Control Expo Risk Assess 28, 107-114.

13. Boon PE, Te Biesebeek JD, Sioen I, et al. (2012) Long-term dietary exposure to lead in young European children: comparing a pan-European approach with a national exposure assessment. Food Addit Contam Part A Chem Anal Control Expo Risk Assess 29, 1701-1715.

14. Yang Y, Wang G \& Pan X (2002) China Food Composition. Beijing: Peking University Medical Press.

15. Su Q, Yu B, He H, et al. (2016) Nut consumption is associated with depressive symptoms among Chinese adults. Depress Anxiety 33, 1065-1072.

16. Lee HC, Chiu HF, Wing YK, et al. (1994) The Zung Self-rating Depression Scale: screening for depression among the Hong Kong Chinese elderly. J Geriatr Psychiatry Neurol 7, 216-220.

17. Xu L, Ren J, Cheng M, et al. (2004) Depressive symptoms and risk factors in Chinese persons with type 2 diabetes. Arch Med Res 35, 301-307.

18. Zung WW (1965) A Self-Rating Depression Scale. Arch Gen Psychiatry 12, 63-70.
19. Yu B, He H, Zhang Q, et al. (2015) Soft drink consumption is associated with depressive symptoms among adults in China. J Affect Disord 172, 422-427.

20. Alberti KG, Eckel RH, Grundy SM, et al. (2009) Harmonizing the metabolic syndrome: a joint interim statement of the International Diabetes Federation Task Force on Epidemiology and Prevention; National Heart, Lung, and Blood Institute; American Heart Association; World Heart Federation; International Atherosclerosis Society; and International Association for the Study of Obesity. Circulation 120, 1640-1645.

21. Craig CL, Marshall AL, Sjostrom M, et al. (2003) International physical activity questionnaire: 12-country reliability and validity. Med Sci Sports Exerc 35, 1381-1395.

22. Andreasson A, Arborelius L, Erlanson-Albertsson C, et al. (2007) A putative role for cytokines in the impaired appetite in depression. Brain Behav Immun 21, 147-152.

23. Cassano P \& Fava M (2002) Depression and public health: an overview. J Psychosom Res 53, 849-857.

24. Patrick L (2006) Lead toxicity, a review of the literature. Part I: exposure, evaluation, and treatment. Altern Med Rev 11, 2-23.

25. Balbus-Kornfeld JM, Stewart W, Bolla KI, et al. (1995) Cumulative exposure to inorganic lead and neurobehavioural test performance in adults: an epidemiological review. Oсcup Environl Med 52, 2-12.

26. Shih RA, Hu H, Weisskopf MG, et al. (2007) Cumulative lead dose and cognitive function in adults: a review of studies that measured both blood lead and bone lead. Environ Health Perspect 115, 483-492.

27. Rhodes D, Spiro A 3rd, Aro A, et al. (2003) Relationship of bone and blood lead levels to psychiatric symptoms: the normative aging study. J Occup Environ Med 45, 1144-1151.

28. Bouchard MF, Bellinger DC, Weuve J, et al. (2009) Blood lead levels and major depressive disorder, panic disorder, and generalized anxiety disorder in US young adults. Arch Gen Psychiatry 66, 1313-1319.

29. Yoon JH \& Ahn YS (2016) The association between blood lead level and clinical mental disorders in fifty thousand leadexposed male workers. J Affect Disord 190, 41-46.

30. Philip AT \& Gerson B (1994) Lead poisoning - part I. Incidence, etiology, and toxicokinetics. Clin Lab Med 14, 423-444.

31. Dunlop BW \& Nemeroff CB (2007) The role of dopamine in the pathophysiology of depression. Arch Gen Psychiatry 64 , 327-337.

32. Kala SV \& Jadhav AL (1995) Region-specific alterations in dopamine and serotonin metabolism in brains of rats exposed to low levels of lead. Neurotoxicology 16, 297-308.

33. Verstraeten SV, Aimo L \& Oteiza PI (2008) Aluminium and lead: molecular mechanisms of brain toxicity. Arch Toxicol 82, 789-802.

34. Cory-Slechta DA, Virgolini MB, Rossi-George A, et al. (2008) Lifetime consequences of combined maternal lead and stress. Basic Clin Pharmacol Toxicol 102, 218-227.

35. Virgolini MB, Chen K, Weston DD, et al. (2005) Interactions of chronic lead exposure and intermittent stress: consequences for brain catecholamine systems and associated behaviors and HPA axis function. Toxicol Sci $\mathbf{8 7}, 469-482$.

36. Eum KD, Nie LH, Schwartz J, et al. (2011) Prospective cohort study of lead exposure and electrocardiographic conduction disturbances in the Department of Veterans Affairs Normative Aging Study. Environ Health Perspect 119, 940-944.

37. Navas-Acien A, Guallar E, Silbergeld EK, et al. (2007) Lead exposure and cardiovascular disease - a systematic review. Environ Health Perspect 115, 472-482. 